\title{
A compact Ge-rich graded-index SiGe platform with broadband low-loss propagation in the mid infrared
}

\author{
Joan Manel Ramírez ${ }^{1}$, Qiankun Liu ${ }^{1}$, Vladyslav Vakarin ${ }^{1}$, Jacopo Frigerio ${ }^{2}$, Andrea Ballabio ${ }^{2}$, Xavier Le \\ Roux $^{1}$, Laurent Vivien ${ }^{1}$, Giovanni Isella ${ }^{2}$, Delphine Marris-Morini ${ }^{1}$ \\ 1. Centre de Nanosciences et de Nanotechnologies, CNRS, Univ. Paris-Sud, Université Paris Saclay, C2N-Orsay, 91405 Orsay cedex, \\ France \\ 2. $\quad$ L-NESS, Dipartimento di Fisica, Politecnico di Milano, Polo di Como, Via Anzani 42, I 22100 Como, Italy \\ Authore-mail address: qiankun.liu@u-psud.fr
}

\begin{abstract}
Ge-rich SiGe platforms with broadband and flat propagation loss of $2-3 \mathrm{~dB} / \mathrm{cm}$ from $\lambda=$ $5.5 \mu \mathrm{m}$ to $8.5 \mu \mathrm{m}$ are demonstrated. Such mid-IR integrated circuits are promising for many application including sensing or telecommunications.

OCIS codes: (190.090) Nonlinear optics; (130.0130) Integrated optics
\end{abstract}

Mid-IR integrated photonics is recently gaining attention due to the wide range of applications foreseen including chemical and biological sensing, thermal imaging, early medical diagnosis or secure communications [1]. Among others, the possibility to use the mature CMOS technology to develop novel photonic integrated circuits exploiting the unique molecular mid-IR absorption bands present in several chemical substances rises as one of the driving forces pushing this research field. This approach would enable ultra-sensitive label-free photonic integrated sensors which could be integrated along with other components in a single photonic chip, hence opening the route towards multifunctional photonic systems. For that, however, a suitable platform capable of integrating several photonic components such as waveguides, Bragg filters, resonators or Mach-Zehnder interferometers should be implemented. Promising mid-IR integrated platforms to carry out this task stand for Ge-on Si, suspended $\mathrm{Si}$ or $\mathrm{SiGe}$ alloys with tailored Ge concentration, among others [2-4]. This latter approach is expected to offer high versatility and broadband transparency [5], and important photonic building blocks such as low-loss waveguides and wideband Mach-Zehnder interferometers have already been demonstrated in the mid infrared [6, 7]. In spite of the progress achieved so far using SiGe alloys, there are still several open questions that require further exploration, such as the disclosure of the platform transparency bandwidth or the investigation of the nonlinear properties in the mid-IR, taking advantage from the absence of two-photon absorption [8].

Thus, we will present our latest results on the development of a novel mid-IR photonic integrated platform based on Ge-rich graded-index SiGe alloys with compact dimensions. Compact waveguides were fabricated in this platform, showing broadband and flat low-loss propagation of $2-3 \mathrm{~dB} / \mathrm{cm}$ over a wavelength range from $\lambda=5.5 \mu \mathrm{m}$ to $8.5 \mu \mathrm{m}$. Moreover, waveguide dispersion engineering was performed to obtain broadband tight mode confinement and flat anomalous condition in the mid-IR [9]. These waveguides show promising features to be used as mid-IR nonlinear optical sources exploiting the third-order nonlinearities for supercontinuum generation [10].

\section{References}

[1] Richard Soref, "Mid-infrared photonics in silicon and germanium”, in Nature Photonics, (Macmillan Publishers Limited, 2010) pp. 495-497

[2] A. Malik, 'Germanium-on-silicon mid-infrared arrayed waveguide grating multiplexers,' IEEE Photonics Technology Letters, 25(18), 18051808 (2013).

[3] Z. Cheng, 'Mid-infrared suspended membrane waveguide and ring resonator on silicon-on-insulator,' IEEE photonics journal, 4(5), 15101519 (2012).

[4] M. Brun, 'Low loss SiGe graded index waveguides for mid-IR applications,' Optics express, 22(1), 508-518 (2014).

[5] J. M. Ramirez, et al. 'Ge-rich SiGe waveguides for mid-infrared photonics,' Proc. Of SPIE 10108 (2017).

[6] JM Ramirez, et al. 'Low-loss Ge-rich Si 0.2 Ge 0.8 waveguides for mid-infrared photonics,' Optics letters 42 (1), 105-108 (2017).

[7] Vladyslav Vakarin et al, "Ultra-wideband Ge-rich silicon germanium integrated Mach Zehnder interferometer for mid infrared spectroscopy" in Optics Letters, (Optical Society of America, 2017) pp. 3482-3485.

[8] F De Leonardis, et al. 'Germanium-on-Silicon Waveguide Engineering for Third Harmonic Generation in the Mid-Infrared,' Journal of Lightwave Technology 33 (24), 5103-5113 (2015)

[9] J.M.Ramirez et al, "Ge-rich graded-index $\mathrm{Si}_{1-\mathrm{x}} \mathrm{Ge}_{\mathrm{x}}$ waveguides with broadband tight mode confinement and flat anomalous dispersion for nonlinear mid-infrared photonics", in Optics Express, (Optical Society of America, 2017) pp. 6561-6567.

[10] S. Serna, et al. 'Nonlinear Properties of Ge-rich Si 1-x Ge x Materials with Different Ge Concentrations,' Sci. Rep. 7(1), 14692 (2017).

\section{Acknowledgment :}

This project has received funding from the European Research Council (ERC) under the European Union's Horizon 2020 research and innovation programme (grant agreement $\mathrm{N}^{\circ} 639107-$-INsPIRE). 